\title{
Transmission of longitudinal wave through micro-porous elastic solid interface
}

\author{
S. K. Tomar ${ }^{1 *}$, Aarti Khurana ${ }^{2}$ \\ ${ }^{I}$ Department of Mathematics, Panjab University, Chandigarh, INDIA \\ ${ }^{2}$ DAV College, Sector-10, Chandigarh, INDIA \\ *Corresponding Author: e-mail: sktomar66@gmail.com, Tel+91-172-2534523, Fax. +91-172-2541132
}

\begin{abstract}
An investigation of reflection and transmission phenomena of plane longitudinal wave from a plane interface between two distinct micropolar porous elastic solid half-spaces in welded contact has been made. Using the method of potentials, the appropriate boundary conditions at the interface are solved to obtain the amplitudes of various reflected and transmitted waves. It has been noticed that these amplitudes are complex valued and their absolute values depend upon the angle of incidence, frequency of the incident wave and on the elastic characteristics of the medium. Numerical computations for a peculiar model are also performed. The variation of modulus of amplitudes of various reflected and transmitted waves against the angle of incidence are computed for obliquely incident longitudinal displacement wave traveling at high frequency as well as at low frequency. At each angle of incidence, the energy balance law has been verified at the interface.
\end{abstract}

Keywords: Micropolar, porous, reflection, longitudinal wave, amplitude, frequency.

\section{Introduction}

A homogeneous isotropic micropolar elastic material is a material characterized by a continuum in which rigid grains of dumb-bell shaped and of infinitesimal size are uniformly distributed in the elastic solid. Eringen (1966, 68) developed the theory of micropolar elasticity which is a subclass of the non-linear theory of simple microelastic solids earlier developed by Eringen and his co-worker (Eringen and Suhubi, 1964; Suhubi and Eringen, 1964) and a generalization of the classical theory of elasticity. The basic difference between the Eringen's theory of micropolar elasticity and that of classical elasticity is the introduction of an independent microrotation vector. Thus, in the theory of micropolar elasticity, the motion in a body is characterized by six degrees of freedom, namely, three of translation and three of rotation, whereas, in classical elasticity theory, the motion is described by displacement vector only and hence, there are three degrees of freedom. The interaction between two parts of a micropolar body is transmitted not only by a force vector but also by a couple resulting in asymmetric force stress tensor and couple stress tensor. The force at a point of a surface element of micropolar elastic solid is completely known by a force stress vector and by a couple stress vector at that point. Parfitt and Eringen (1969) have shown that there can exist four waves in a micropolar elastic material, two of which disappear below a critical frequency dependent upon the property of the medium.

Material having small distributed voids containing nothing of physical significance is called porous material. Cowin and his coworker (Nunziato and Cowin, 1979; Cowin and Nunziato, 1983) developed non-linear and linear theories of elastic material with voids. In linear theory of elastic material with voids, the change in void volume fraction and the strain are taken as independent kinematic variables. Due to the introduction of this new variable, there exist a higher order stress called equilibrated force, intrinsic and extrinsic body forces and equilibrated inertia (see Goodman and Cowin, 1972; Cowin and Goodman, 1976). Puri and Cowin (1985) analyzed the frequency equation of plane harmonic waves in a linear elastic material with voids. They showed that there exist two dilational waves in porous elastic material with voids, one of them is predominantly the dilational wave of classical linear elasticity and the other is predominantly a wave carrying a change in the void volume fraction. Both these waves are found to attenuate in their direction of propagation. At large frequencies, the predominantly elastic wave propagates with the classical elastic dilational wave speed, but at low frequency it propagates at a speed less than the classical wave speed. Numerous problems of waves and vibrations concerning the micropolar elasticity and material with voids have been attempted by many researchers in the past, e.g., Chandersekhariah (1987), Wright (1998), Iesan and Nappa (2003), Golamhossen (2000), Dey et al. (2003), Midya (2004), Singh and Kumar (1998), Tomar and Gogna (1995a, 95b) among several others. The problems of reflection and refraction of elastic waves from a plane interface are very 
important in the inspection of geological materials, rocks and manufactured materials. Several classical problems addressing the reflection and refraction of elastic waves from interfaces between elastic media have been studied by considering different models and incorporating numerous possible parameters in them. Ciarletta and Sumbatyan (2003) discussed the reflection of obliquely incident plane transverse shear waves from the free boundary of a elastic half-space with voids. They showed that only the transverse shear wave can propagate without attenuation after having been reflected from the free boundary surface. They have also obtained the reflection coefficients asymptotically in closed form for high and low frequency cases. Recently, Tomar and Singh (2005) investigated the transmission of longitudinal waves through a plane interface between two dissimilar porous elastic solid half-spaces.

Recently, Tomar and Singh (2006) have discussed the propagation of plane waves in an infinite micropolar porous elastic solid. They encountered that there may exist five waves in an infinite micropolar porous elastic solid comprising of three longitudinal waves and two sets of coupled transverse waves. The three longitudinal waves are: a longitudinal microrotational wave travelling independently and not influenced with the presence of voids, a longitudinal displacement wave and a longitudinal volume fractional wave carrying the change in void volume fraction. The longitudinal microrotational wave and two sets of coupled transverse waves are same as earlier encountered by Parfitt and Eringen (1969) in micropolar elastic solid. They have also studied the reflection phenomenon of these plane waves from a free plane boundary of a micropolar porous elastic solid half-space.

In this paper, we have discussed the phenomena of reflection and transmission of a longitudinal displacement wave striking obliquely at a plane interface between two distinct micropolar porous elastic solid half-spaces in perfect contact. The appropriate boundary conditions at the interface are formulated and the amplitudes of various reflected and transmitted waves are obtained. It has been noticed that the amplitudes are complex valued and their absolute values depend upon the angle of incidence, frequency of the incident wave and on the elastic parameters. The variation of modulus of amplitudes against the angle of incidence of longitudinal displacement wave impinging at high frequency and at low frequency are also depicted graphically. The analytical expressions of energy ratios of various reflected and transmitted waves are also presented. It has been observed that there is no dissipation of energy at the interface during transmission.

\section{Basic equations and relations}

In the absence of body force density and body couple density, the field equations in micropolar elastic material with voids are given by (see Iesan, 1985)

$$
\begin{gathered}
(\lambda+2 \mu+K) \nabla \nabla \cdot u-(\mu+K) \nabla \times \nabla \times u+K \nabla \times \Phi+\beta^{*} \nabla \psi=\rho \ddot{u}, \\
(\alpha+\beta+\gamma) \nabla \nabla \cdot \Phi-\gamma \nabla \times \nabla \times \Phi+K \nabla \times u-2 K \Phi=\rho J \ddot{\Phi}, \\
\alpha^{*} \nabla^{2} \psi-\xi^{*} \psi-\omega^{*} \psi-\beta^{*} \nabla \cdot u=\rho K^{*} \ddot{\Psi},
\end{gathered}
$$

where $\lambda$ and $\mu$ are Lame's constants; $K, \alpha, \beta$ and $\gamma$ are the elastic constants of micropolarity; $\alpha^{*}, \beta^{*}, \xi^{*}, \omega^{*}$ and $K^{*}$ are the elastic constants due to the presence of voids; $u(x, t)$ and $\Phi(x, t)$ are the displacement and microrotation vectors, respectively; $\psi$ is the change in void volume fraction from that of in the reference state; $J$ is micro-inertia and $\rho$ is the density of the medium. The superposed dots on the right hand side of these equations represent the temporal derivatives.

For time harmonic plane wave propagation (i.e., $\propto \exp \{-i \omega t\}$ ), the equations of motion (1) - (3) reduce to

$$
\begin{gathered}
\left(c_{1}^{2}+c_{3}^{2}\right) \nabla \nabla \cdot u-\left(c_{2}^{2}+c_{3}^{2}\right) \nabla \times \nabla \times u+c_{3}^{2} \nabla \times \Phi+c_{6}^{2} \nabla \psi+\omega^{2} u=0, \\
\left(c_{4}^{2}+c_{5}^{2}\right) \nabla \nabla \cdot \Phi-c_{4}^{2} \nabla \times \nabla \times \Phi+\omega_{0}^{2} \nabla \times u-2 \omega_{0}^{2} \Phi+\omega^{2} \Phi=0, \\
\left(\alpha^{*} \nabla^{2}-\xi^{*}+i \omega^{*} \omega+\rho K^{*} \omega^{2}\right) \psi-\beta^{*} \nabla \cdot u=0,
\end{gathered}
$$

where

$$
c_{1}^{2}=\frac{\lambda+2 \mu}{\rho}, c_{2}^{2}=\frac{\mu}{\rho}, c_{3}^{2}=\frac{K}{\rho}, c_{4}^{2}=\frac{\gamma}{\rho J}, c_{5}^{2}=\frac{\alpha+\beta}{\rho J}, c_{6}^{2}=\frac{\beta^{*}}{\rho} \text { and } \omega_{0}^{2}=\frac{K}{\rho J} .
$$

The constitutive relations for the micropolar porous elastic solid are given by (see Iesan, 1985)

$$
\begin{gathered}
t_{i j}=\lambda u_{k, k} \delta_{i j}+\mu\left(u_{i, j}+u_{j, i}\right)+K\left(u_{j, i}-\varepsilon_{i j k} \phi_{k}\right)+\delta_{i j} \beta^{*} \psi, \\
m_{i j}=\alpha \phi_{k, k} \delta_{i j}+\beta \phi_{i, j}+\gamma \phi_{j, i}, \\
h_{i}=\alpha^{*} \psi_{, i},
\end{gathered}
$$

where $t_{i j}, m_{i j}$ and $h_{i}$ are the force stress tensor, couple stress tensor and equilibrated force vector, respectively. 
Introducing the scalar potentials $q$ and $\xi$, the vector potentials $U$ and $\Pi$, through the Helmholtz's decomposition of vectors as

$$
u=\nabla q+\nabla \times U, \quad \Phi=\nabla \xi+\nabla \times \Pi, \quad \nabla \cdot U=\nabla \cdot \Pi=0,
$$

and employing these relations into equations of motion (4) - (6), we obtain the following system of equations

$$
\begin{aligned}
& {\left[\left(c_{1}^{2}+c_{3}^{2}\right) \nabla^{2}+\omega^{2}\right] q+c_{6}^{2} \psi=0,} \\
& {\left[\left(c_{2}^{2}+c_{3}^{2}\right) \nabla^{2}+\omega^{2}\right] U+c_{3}^{2} \nabla \times \Pi=0,} \\
& {\left[\left(c_{4}^{2}+c_{5}^{2}\right) \nabla^{2}-2 \omega_{0}^{2}+\omega^{2}\right] \xi=0,} \\
& {\left[c_{4}^{2} \nabla^{2}-2 \omega_{0}^{2}+\omega^{2}\right] \Pi+\omega_{0}^{2} \nabla \times U=0,} \\
& \left(\alpha^{*} \nabla^{2}-\xi^{*}+i \omega \omega^{*}+\rho K^{*} \omega^{2}\right) \psi-\beta^{*} \nabla^{2} q=0 .
\end{aligned}
$$

Following the procedure adopted by Tomar and Singh (2006) for plane waves advancing along the positive direction of a unit vector, we can obtain the dispersion relations giving the phase speeds of an independent longitudinal microrotational wave and two sets of coupled transverse waves along with the following dispersion equation giving the phase speeds of two longitudinal waves

$$
\left(k^{2}-\frac{\omega^{2}}{S^{2}}\right)\left(k^{2}-\frac{\omega^{2}}{T^{2}}+\frac{1}{l_{2}^{2}}-\frac{i \omega \omega^{*}}{\alpha^{*}}\right)-\frac{H^{*}}{l_{1}^{2}} k^{2}=0,
$$

where

$$
S^{2}=c_{1}^{2}+c_{3}^{2}, \quad T^{2}=\frac{\alpha^{*}}{\rho K^{*}}, \quad l_{2}^{2}=\frac{\alpha^{*}}{\xi^{*}}, \quad l_{1}^{2}=\frac{\alpha^{*}}{\beta^{*}} \text { and } H^{*}=\frac{\beta^{*}}{\lambda+2 \mu+K} .
$$

The quantity $S$ is the speed of propagation of longitudinal displacement wave (see Parfitt and Eringen, 1969) and $H^{*}$ is a coupling dimensionless number similar to that introduced earlier by Puri and Cowin (1985) and reduces to it in the absence of micropolarity. Also, equation (17) can be written as

$$
\left(k^{2}-\frac{\omega^{2}}{S^{2}}\right)\left(k^{2}-\frac{\omega^{2}}{T^{2}}+\frac{1}{l_{2}^{2}}-\frac{i \omega \omega^{*}}{\alpha^{*}}\right)-\frac{N^{*}}{l_{2}^{2}} k^{2}=0
$$

where $N^{*}=\frac{l_{2}^{2} H^{*}}{l_{1}^{2}}$ and $0<N^{*}<1$.

Tomar and Singh (2006) have shown that the two roots of bi-quadratic equation (18) are given by

and

$$
k_{1}=\frac{\omega}{S}, k_{2}=\frac{\omega}{T}+\frac{i \omega^{*} T}{2 \alpha^{*}}, \quad \text { for high frequency }\left(l_{2} \omega>>1\right),
$$

$$
k_{1}=\frac{\omega}{S \sqrt{1-N^{*}}}, \quad k_{2}=\frac{\omega}{c_{4}^{*}}+\frac{i \sqrt{1-N^{*}}}{l_{2}}, \quad \text { for low frequency }\left(l_{2} \omega<<1\right),
$$

where $c_{4}^{*}=\frac{2 \alpha^{*} \sqrt{1-N^{*}}}{\omega^{*} l_{2}}$.

\section{Reflection/refraction}

We shall discuss the reflection and transmission phenomena of a longitudinal displacement wave incident obliquely at the plane interface between two distinct micropolar porous elastic solid half-spaces in perfect contact. 


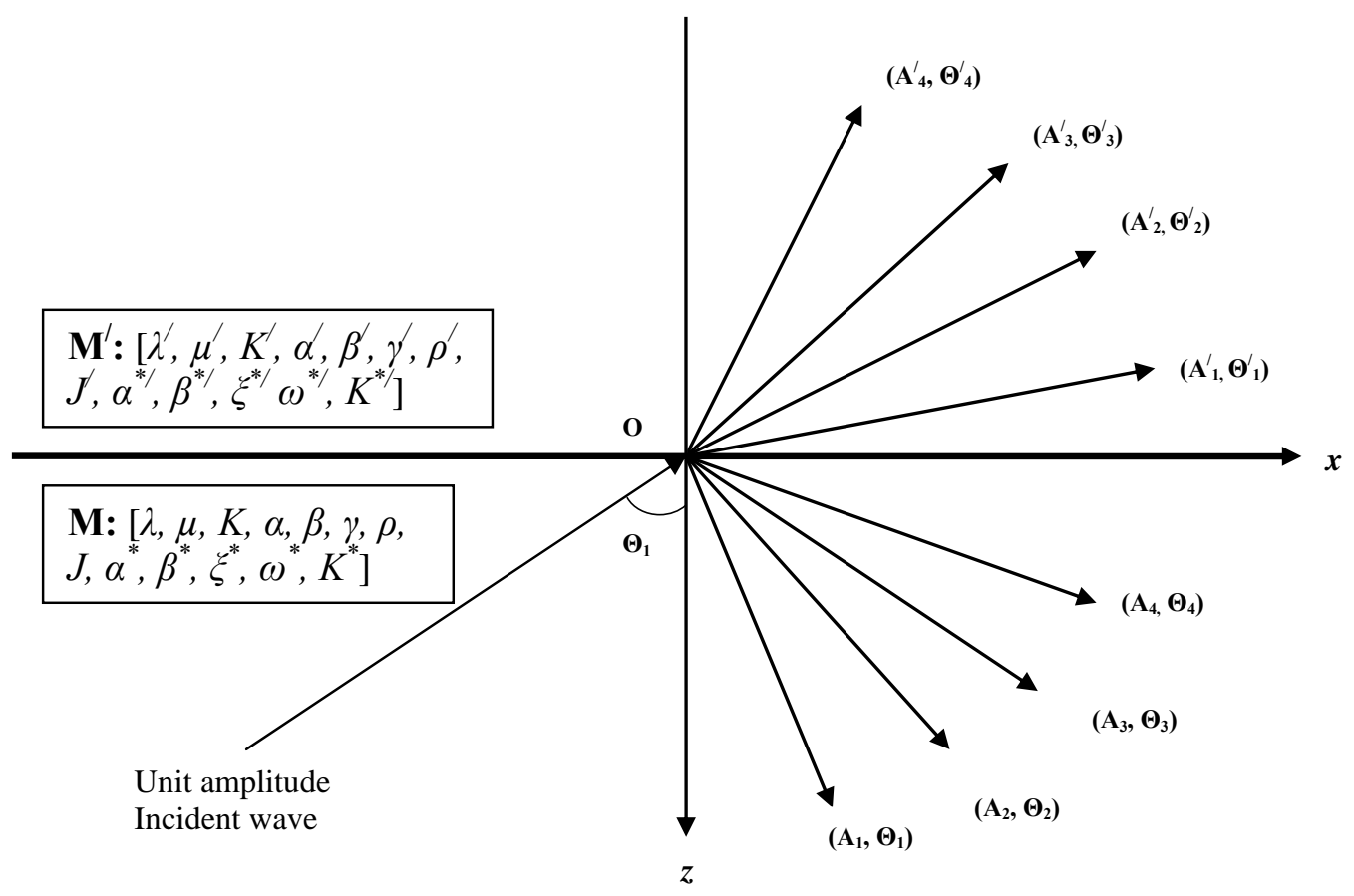

Figure 1: Schematic diagram of the problem.

Let the interface be along $x$-axis and $z$-axis be taken along the direction pointing vertically downward. We take the lower half-space as medium $M[z>0]$ and denote the elastic constants and density in this medium by $\lambda, \mu, K, \alpha, \beta, \gamma, J, \alpha^{*}, \beta^{*}, \xi^{*}, \omega^{*}, K^{*}$ and $\rho$, while the upper half-space as medium $M^{\prime}[z<0]$ and the corresponding elastic parameters therein are denoted by $\lambda^{\prime}, \mu^{\prime}, K^{\prime}, \alpha^{\prime}, \beta^{\prime}, \gamma^{\prime}, J^{\prime}, \alpha^{* \prime}, \beta^{* \prime}, \xi^{* \prime}, \omega^{* \prime}, K^{* \prime}$ and $\rho^{\prime}$. The complete geometry of the problem is shown in Figure 1.

We consider a two-dimensional problem in $x-z$ plane by taking

$$
u=\left(u_{1}, 0, u_{3}\right), \Phi=\left(0, \phi_{2}, 0\right), \psi=\psi(x, z) .
$$

Let a unit amplitude plane longitudinal displacement wave propagating with wavenumber $k_{1}$ and making an angle $\theta_{1}$ with the normal be incident at the interface $z=0$. We postulate the appearance of following reflected and transmitted waves to satisfy the boundary conditions at the interface:

\section{Reflected waves:}

(i) A longitudinal displacement wave of amplitude $A_{1}$ making angle $\theta_{1}$ with the normal,

(ii) A longitudinal volume fractional wave of amplitude $A_{2}$ making an angle $\theta_{2}$ with the normal,

(iii) Two sets of coupled transverse waves of amplitudes $A_{3,4}$ propagating with speeds $V_{3,4}$ and making angles $\theta_{3,4}$ with the normal.

\section{Transmitted waves:}

(i) A longitudinal displacement wave of amplitude $A_{1}^{\prime}$ making angle $\theta_{1}^{\prime}$ with the normal.

(ii) A longitudinal volume fractional wave of amplitude $A_{2}^{\prime}$ making an angle $\theta_{2}^{\prime}$ with the normal.

(iii) Two sets of coupled transverse waves of amplitudes $A_{3,4}^{\prime}$ propagating with speeds $V_{3,4}^{\prime}$ and making angles $\theta_{3,4}^{\prime}$ with the normal.

The expressions of speeds $V_{3,4}$ are given earlier in Tomar and Singh (2006). The expressions of speeds $V_{3,4}^{\prime}$ are similar to the expression of speeds $V_{3,4}$, with appropriate dashes.

The full structure of the wave field consisting of incident, reflected and transmitted waves are given as: In medium $M$, 


$$
q=P_{1}^{-}+\sum_{i=1,2} A_{i} P_{i}^{+}, \quad U_{2}=\sum_{i=3,4} A_{i} P_{i}^{+}, \quad \phi_{2}=\sum_{i=3,4} \eta_{i} A_{i} P_{i}^{+},
$$

and in medium $M^{\prime}$,

$$
q^{\prime}=\sum_{i=1,2} A_{i}^{\prime} P_{i}^{\prime^{-}}, U_{2}^{\prime}=\sum_{i=3,4} A_{i}^{\prime} P_{i}^{\prime^{-}}, \quad \phi_{2}=\sum_{i=3,4} \eta_{i}^{\prime} A_{i}^{\prime} P_{i}^{\prime^{-}},
$$

where $P_{1}^{-}=\exp \left\{i k_{1}\left(\sin \theta_{1} x-\cos \theta_{1} z\right)-i \omega_{1} t\right\}, P_{i}^{+}=\exp \left\{i k_{i}\left(\sin \theta_{i} x+\cos \theta_{i} z\right)-i \omega_{i} t\right\}$,

$P_{i}^{{ }^{-}}=\exp \left\{i k_{i}^{\prime}\left(\sin \theta_{i}^{\prime} x-\cos \theta_{i}^{\prime} z\right)-i \omega_{i}^{\prime} t\right\}, \omega_{i}\left(=k_{i} V_{i}\right)$ and $\omega_{i}^{\prime}\left(=k_{i}^{\prime} V_{i}^{\prime}\right)$ are the frequencies of the respective waves. The amplitudes $A_{i}$ and $A_{i}^{\prime}(i=1,2,3,4)$ can be determined using appropriate boundary conditions at the interface $z=0$. The quantities $\eta_{3,4}$ are the coupling parameters between $U_{2}$ (the $y$-component of vector $U$ ) and $\phi_{2}$, are given already by Parfitt and Eringen (1969) and can be rewritten as follows

$$
\eta_{3,4}=\omega_{0}^{2}\left[V_{3,4}^{2}-2 \frac{\omega_{0}^{2}}{k_{3,4}^{2}}-c_{4}^{2}\right]^{-1} .
$$

The quantities $\eta_{3,4}^{\prime}$ are the coupling parameters between $U_{2}^{\prime}$ and $\phi_{2}^{\prime}$ and their expressions are similar to $\eta_{3,4}$ by putting appropriate dashes.

Using equations from (11) into equations (8) - (10), we can write the requisite components of stresses and displacements into potential form. The requisite components of stresses are given by

$$
\begin{aligned}
& t_{z z}=(\lambda+2 \mu+K) q_{, z z}+(2 \mu+K) U_{2, x z}+\lambda q_{, x x}+\beta^{*} \psi, \\
& t_{z x}=(2 \mu+K) q_{, x z}-(\mu+K) U_{2, z z}+\mu U_{2, x x}-K \phi_{2}, \\
& m_{z y}=\gamma \phi_{2, z}, \\
& h_{z}=\alpha^{*} \psi_{, z},
\end{aligned}
$$

and the requisite components of displacements are given by

$$
u_{1}=q_{, x}-U_{2, z}, \quad u_{3}=q_{, z}+U_{2, x}
$$

The expressions of stresses $t_{z z}^{\prime}, t_{z x}^{\prime}, m_{z y}^{\prime}, h_{z}^{\prime}$ and displacements $u_{1}^{\prime}$ and $u_{3}^{\prime}$ in medium $M^{\prime}$ can be written similarly by putting appropriate dashes, e.g., $m_{z y}^{\prime}=\gamma^{\prime} \phi_{2, z}^{\prime}$ and $u_{1}^{\prime}=q_{, x}^{\prime}-U_{2, z}^{\prime}$, etc. The comma in the subscript denotes the spatial derivative.

At the interface between two micropolar porous elastic solid half-spaces, the appropriate mechanical boundary conditions are: (i) continuity of force stresses, (ii) continuity of couple stresses, (iii) continuity of equilibrated force vector, (iv) continuity of displacement, microrotation and change in void volume fraction.

Mathematically, these boundary conditions can be written as:

At the interface $z=0$,

$$
\begin{array}{llll}
t_{z z}=t_{z z}^{\prime}, & t_{z x}=t_{z x}^{\prime}, & m_{z y}=m_{z y}^{\prime}, & h_{z}=h_{z}^{\prime}, \\
u_{1}=u_{1}^{\prime}, & u_{3}=u_{3}^{\prime}, & \phi_{2}=\phi_{2}^{\prime}, & \psi=\psi^{\prime} .
\end{array}
$$

Using constitutive relations given in (22) and the expressions of potentials $q, U_{2}, \phi_{2}, q^{\prime}, U_{2}^{\prime}$ and $\phi_{2}^{\prime}$ from equations (20) and (21) into the equations in (23), we obtain the following system of eight simultaneous non-homogeneous equations after employing the Snell's law given by

$$
k_{i} \sin \theta_{i}=k_{i}^{\prime} \sin \theta_{i}^{\prime}=k_{1} \sin \theta_{1},(i=1,2,3,4)
$$

and assuming that all frequencies are same at the interface, as follows 


$$
\begin{aligned}
& (2 \mu+K)\left[\left(k_{1}^{2} \sin ^{2} \theta_{1}-\zeta_{1}^{2}\right)+\sum_{i=1,2}\left(k_{i}^{2} \sin ^{2} \theta_{i}-\zeta_{1}^{2}\right) A_{i}-\sum_{i=3,4} k_{i}^{2} \sin \theta_{i} \cos \theta_{i} A_{i}\right] \\
& =\left(2 \mu^{\prime}+K^{\prime}\right)\left[\sum_{i=1,2}\left(k_{i}^{\prime 2} \sin ^{2} \theta_{i}^{\prime}-\zeta_{1}^{\prime 2}\right) A_{i}^{\prime}-\sum_{i=3,4} k_{i}^{\prime 2} \sin \theta_{i}^{\prime} \cos \theta_{i}^{\prime} A_{i}^{\prime}\right], \\
& (2 \mu+K)\left[k_{1}^{2} \sin \theta_{1} \cos \theta_{1}-\sum_{i=1,2} k_{i}^{2} \sin \theta_{i} \cos \theta_{i} A_{i}\right]+\sum_{i=3,4}\left[\mu \cos 2 \theta_{i}+K \cos ^{2} \theta_{i}-\frac{K \eta_{i}}{k_{i}^{2}}\right] k_{i}^{2} A_{i} \\
& =\left(2 \mu^{\prime}+K^{\prime}\right) \sum_{i=1,2} k_{i}^{\prime 2} \sin \theta_{i}^{\prime} \cos \theta_{i}^{\prime} A_{i}^{\prime}+\sum_{i=3,4}\left[\mu^{\prime} \cos 2 \theta_{i}^{\prime}+K^{\prime} \cos ^{2} \theta_{i}^{\prime}-\frac{K^{\prime} \eta_{i}^{\prime}}{k_{i}^{\prime 2}}\right] k_{i}^{\prime 2} A_{i}^{\prime}, \\
& \gamma \sum_{i=3,4} \eta_{i} k_{i} \cos \theta_{i} A_{i}+\gamma^{\prime} \sum_{i=3,4} \eta_{i}^{\prime} k_{i}^{\prime} \cos \theta_{i}^{\prime} A_{i}^{\prime}=0, \\
& \frac{\alpha^{*}(\lambda+2 \mu+K)}{\beta^{*}}\left[\left(k_{1}^{2}-\zeta_{2}^{2}\right) k_{1} \cos \theta_{1}+\sum_{i=1,2}\left(\zeta_{2}^{2}-k_{i}^{2}\right) k_{i} \cos \theta_{i} A_{i}\right] \\
& =\frac{\alpha^{* \prime}\left(\lambda^{\prime}+2 \mu^{\prime}+K^{\prime}\right)}{\beta^{* \prime}} \sum_{i=1,2}\left(k_{i}^{\prime 2}-\zeta_{2}^{\prime 2}\right) k_{i}^{\prime} \cos \theta_{i}^{\prime} A_{i}^{\prime}, \\
& k_{1} \sin \theta_{1}+\sum_{i=1,2} k_{i} \sin \theta_{i} A_{i}-\sum_{i=3,4} k_{i} \cos \theta_{i} A_{i}=\sum_{i=1,2} k_{i}^{\prime} \sin \theta_{i}^{\prime} A_{i}^{\prime}+\sum_{i=3,4} k_{i}^{\prime} \cos \theta_{i}^{\prime} A_{i}^{\prime}, \\
& k_{1} \cos \theta_{1}-\sum_{i=1,2} k_{i} \cos \theta_{i} A_{i}-\sum_{i=3,4} k_{i} \sin \theta_{i} A_{i}=\sum_{i=1,2} k_{i}^{\prime} \cos \theta_{i}^{\prime} A_{i}^{\prime}-\sum_{i=3,4} k_{i}^{\prime} \sin \theta_{i}^{\prime} A_{i}^{\prime} \text {, } \\
& \sum_{i=3,4} \eta_{i} A_{i}-\sum_{i=3,4} \eta_{i}^{\prime} A_{i}^{\prime}=0 \\
& \frac{(\lambda+2 \mu+K)}{\beta^{*}}\left[\left(\zeta_{2}^{2}-k_{1}^{2}\right)+\sum_{i=1,2}\left(\zeta_{2}^{2}-k_{i}^{2}\right) A_{i}\right]=\frac{\left(\lambda^{\prime}+2 \mu^{\prime}+K^{\prime}\right)}{\beta^{* \prime}} \sum_{i=1,2}\left(\zeta_{2}^{\prime 2}-k_{i}^{\prime 2}\right) A_{i}^{\prime},
\end{aligned}
$$

where

$$
\zeta_{1}^{2}=\frac{\omega^{2}}{c_{3}^{2}+2 c_{2}^{2}}, \quad \zeta_{2}^{2}=\frac{\omega^{2}}{c_{1}^{2}+c_{3}^{2}}, \quad \zeta_{1}^{\prime 2}=\frac{\omega^{2}}{c_{3}^{\prime 2}+2 c_{2}^{\prime 2}} \text { and } \zeta_{2}^{\prime 2}=\frac{\omega^{2}}{c_{1}^{\prime 2}+c_{3}^{\prime 2}} .
$$

These equations enable us to provide the formulae for the amplitudes $A_{i}$ and $A_{i}^{\prime}(i=1,2,3,4)$ of various reflected and transmitted waves at the plane interface $z=0$.

\section{Energy partitioning}

Now, we consider the energy partitioning between various reflected and refracted waves at the interface $z=0$. The rate of energy transmission per unit area is given by

$$
P^{*}=t_{z z} \dot{u}_{3}+t_{z x} \dot{u}_{1}+m_{z y} \dot{\phi}_{2}
$$

The energy $E_{\text {inc }}^{l}$ of incident longitudinal displacement wave is given by

$$
E_{i n c}^{l}=\rho \omega^{3} k_{1} \cos \theta_{1} \exp \left\{2 i\left[k_{1}\left(\sin \theta_{1} x-\cos \theta_{1} z\right)-\omega t\right]\right\}
$$

The energy ratios $E_{i}(i=1,2,3, \ldots, 8)$ of reflected longitudinal displacement wave, reflected longitudinal volume fractional wave, reflected sets of coupled transverse waves, refracted longitudinal displacement wave, refracted longitudinal volume fractional wave, refracted sets of coupled transverse waves to the incident wave are given by

$$
E_{1}=\left|A_{1}\right|^{2}
$$




$$
\begin{aligned}
& E_{2}=\frac{k_{2} \cos \theta_{2}}{k_{1} \cos \theta_{1}}\left|A_{2}\right|^{2}, \\
& E_{3}=\frac{k_{3}^{3} \cos \theta_{3}}{\rho \omega^{2} k_{1} \cos \theta_{1}}\left[\mu+K-\frac{\eta_{3}}{k_{3}^{2}}\left(\gamma \eta_{3}+K\right)\right]\left|A_{3}\right|^{2}, \\
& E_{4}=\frac{k_{4}^{3} \cos \theta_{4}}{\rho \omega^{2} k_{1} \cos \theta_{1}}\left[\mu+K-\frac{\eta_{4}}{k_{4}^{2}}\left(\gamma \eta_{4}+K\right)\right]\left|A_{4}\right|^{2}, \\
& E_{5}=\frac{\rho^{\prime} k_{1}^{\prime} \cos \theta_{1}^{\prime}}{\rho k_{1} \cos \theta_{1}}\left|A_{1}^{\prime}\right|^{2}, \\
& E_{6}=\frac{\rho^{\prime} k_{2}^{\prime} \cos \theta_{2}^{\prime}}{\rho k_{1} \cos \theta_{1}}\left|A_{2}^{\prime}\right|^{2}, \\
& E_{7}=\frac{k_{3}^{\prime 3} \cos \theta_{3}^{\prime}}{\rho \omega^{2} k_{1} \cos \theta_{1}}\left[\mu^{\prime}+K^{\prime}-\frac{\eta_{3}^{\prime}}{k_{3}^{\prime 2}}\left(\gamma^{\prime} \eta_{3}^{\prime}+K^{\prime}\right)\right]\left|A_{3}^{\prime}\right|^{2}, \\
& E_{8}=\frac{k_{4}^{\prime 3} \cos \theta_{4}^{\prime}}{\rho \omega^{2} k_{1} \cos \theta_{1}}\left[\mu^{\prime}+K^{\prime}-\frac{\eta_{4}^{\prime}}{k_{4}^{\prime 2}}\left(\gamma^{\prime} \eta_{4}^{\prime}+K^{\prime}\right)\right]\left|A_{4}^{\prime}\right|^{2} .
\end{aligned}
$$

\section{Numerical discussion}

In order to study the problem in greater detail, we shall solve equations (25) - (32) numerically to find the nature of dependence of these amplitudes of various reflected and transmitted waves with angle of incidence for low as well as for high frequency. For this purpose, we have taken the following numerical values of relevant physical parameters occurring in this problem.

\begin{tabular}{|c|c|c|c|c|}
\hline Symbol & Value & Symbol & Value & Unit \\
\hline$\lambda$ & $7.55 \times 10^{11}$ & $\lambda^{\prime}$ & $5.5 \times 10^{11}$ & dyne $/ \mathrm{cm}^{2}$ \\
\hline$\mu$ & $6.19 \times 10^{11}$ & $\mu^{\prime}$ & $2.14 \times 10^{11}$ & dyne $/ \mathrm{cm}^{2}$ \\
\hline$K$ & $0.145 \times 10^{11}$ & $K^{\prime}$ & $0.129 \times 10^{11}$ & dyne $/ \mathrm{cm}^{2}$ \\
\hline$\gamma$ & $2.86 \times 10^{11}$ & $\gamma^{\prime}$ & $1.88 \times 10^{11}$ & dyne \\
\hline$J$ & 0.0212 & $J^{\prime}$ & 0.0166 & $\mathrm{~cm}^{2}$ \\
\hline$\rho$ & 2.6 & $\rho^{\prime}$ & 2.2 & $\mathrm{gm} / \mathrm{cm}^{3}$ \\
\hline$\xi^{*}$ & $12 \times 10^{11}$ & $\xi^{*}$ & $10 \times 10^{11}$ & dyne $/ \mathrm{cm}^{2}$ \\
\hline$\beta^{*}$ & $10 \times 10^{11}$ & $\beta^{*}$ & $8 \times 10^{11}$ & dyne $/ \mathrm{cm}^{2}$ \\
\hline$\omega^{*}$ & $0.02 \times 10^{11}$ & $\omega^{*}$ & $0.01 \times 10^{11}$ & dyne s/cm ${ }^{2}$ \\
\hline$\alpha^{*}$ & $0.004 \times 10^{11}$ & $\alpha^{*}$ & $0.002 \times 10^{11}$ & dyne \\
\hline
\end{tabular}

Matrix inversion method is used to solve equations (25) - (32) for amplitudes of various reflected and transmitted waves. Figures 2 and 3 depict the variation of modulus of amplitudes of various reflected and transmitted waves with respect to the angle of incidence, when a longitudinal displacement wave is incident obliquely at the interface with frequency $\omega=10 \mathrm{rad} \mathrm{s}^{-1}$, (i.e., when $l_{2} \omega<<1$ ). We found that all the amplitudes are complex valued at each angle of incidence. 


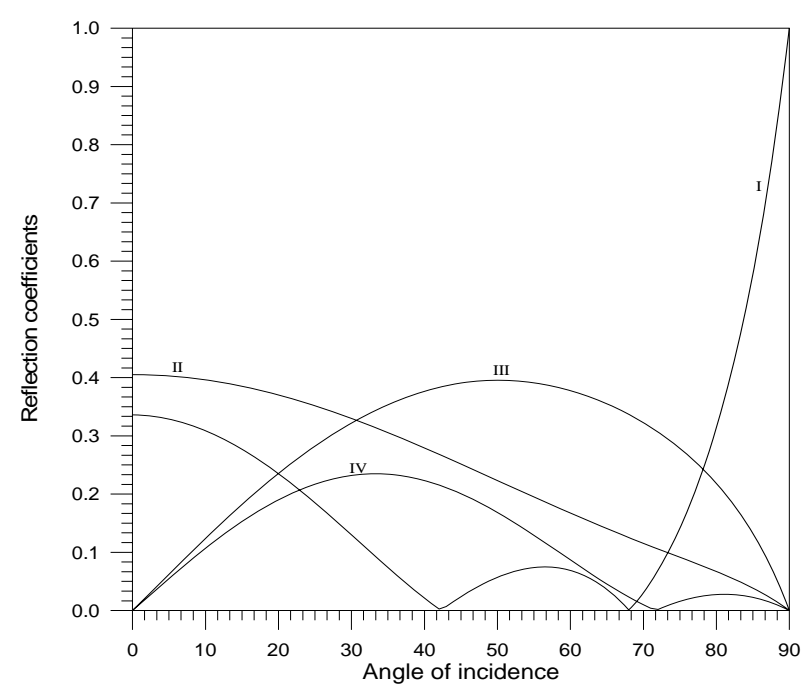

Figure 2: (Low frequency case) Variation of reflection Figure 3: (Low frequency case) Variation of refraction coefficients with the angle of incidence (Curve-I: $\left|A_{1}\right|$, CurveII: $\left|A_{2}\right| \times 10^{13}$, Curve-III: $\left|A_{3}\right| \times 10^{15}$, Curve -IV: $\left.\left|A_{4}\right|\right)$

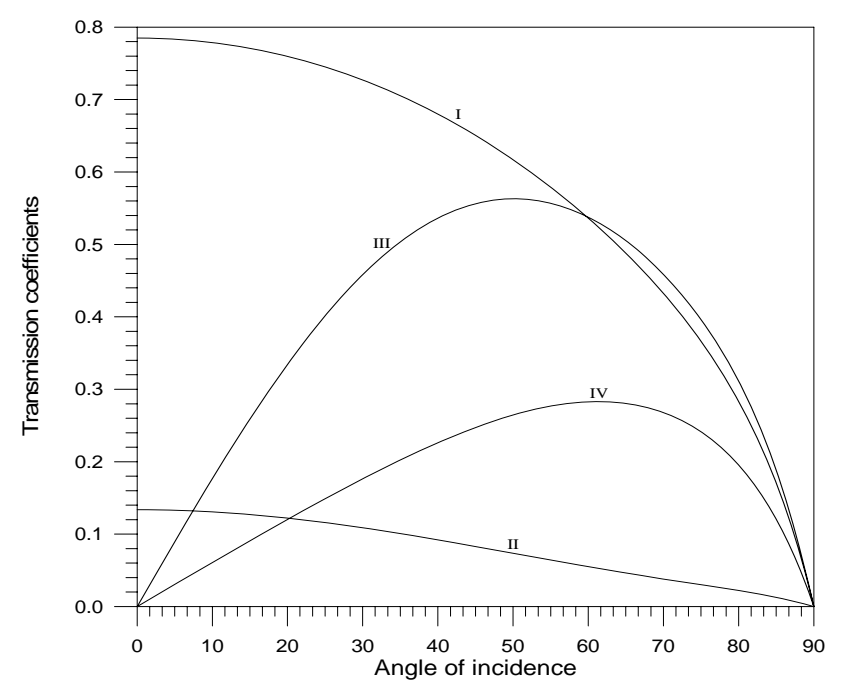
coefficients with angle of incidence (Curve-I: $\left|A_{1}^{\prime}\right|$, Curve-II: $\left|A_{2}^{\prime}\right| \times 10^{12}$, Curve-III: $\left|A_{3}^{\prime}\right| \times 10^{15}$, Curve -IV: $\left|A_{4}^{\prime}\right|$ )

In Figure 2, we have plotted the modulus of amplitudes of various reflected waves against the angle of incidence. It has been noticed that the amplitude $\left|A_{2}\right|$ of the reflected volume fractional wave and the amplitude $\left|A_{3}\right|$ corresponding to the set of coupled transverse waves propagating with speed $V_{3}$ are quite small in comparison to the amplitudes $\left|A_{1}\right|$ and $\left|A_{4}\right|$. Therefore, these amplitudes $\left|A_{2}\right|$ and $\left|A_{3}\right|$ have been plotted after magnifying their original values by the factors of $10^{13}$ and $10^{15}$, respectively. The amplitude $\left|A_{1}\right|$ begins with the value 0.3358 near the normal incidence, it decreases till $\theta_{1}=42^{0}$, and afterwards it increases very slowly upto $\theta_{1}=56^{\circ}$. Beyond $\theta_{1}=56^{\circ}$, the amplitude $\left|A_{1}\right|$ decreases very smoothly till $\theta_{1}=68^{\circ}$ and thereafter it increases frequently and attains the maximum value, i.e., unity at the grazing incidence. The amplitude $\left|A_{2}\right|$ decreases throughout in the entire range with increase of $\theta_{1}$. The amplitudes $\left|A_{3}\right|$ and $\left|A_{4}\right|$ begin with value zero at normal incidence. The value of amplitude $\left|A_{3}\right|$ increases with increase in the angle of incidence till $\theta_{1}=50^{\circ}$ and then, it gradually decreases to the value zero at grazing incidence. The value of amplitude $\left|A_{4}\right|$ increases slowly with increase of angle of incidence till $\theta_{1}=33^{0}$, then its value decreases till $\theta_{1}=72^{0}$. After $\theta_{1}=72^{0}$, the amplitude $\left|A_{4}\right|$ again increases very slowly with angle of incidence till a certain angle and thereafter it decreases and vanishes at $\theta_{1}=90^{\circ}$.

In Figure 3, we have shown the variation of modulus values of amplitudes of various refracted waves with the angle of incidence. We observed that the amplitudes $\left|A_{1}^{\prime}\right|$ and $\left|A_{2}^{\prime}\right|$ of transmitted longitudinal displacement wave and volume fractional wave attain their maximum values at normal incidence and then their values decrease gradually to zero at the grazing incidence. Both the amplitudes $\left|A_{3}^{\prime}\right|$ and $\left|A_{4}^{\prime}\right|$ have value zero near normal incidence, then both of them increase with increase in angle of incidence and attain their maximum values near $\theta_{1}=60^{\circ}$. Thereafter, they decrease and vanish at grazing incidence. However, the maximum value of $\left|A_{4}^{\prime}\right|$ is greater than the maximum value of $\left|A_{3}^{\prime}\right|$. Also, it is noted that the modulus of $\left|A_{1}^{\prime}\right|$ corresponding to the transmitted longitudinal displacement wave propagating with velocity $V_{1}^{\prime}$ is contributing significantly as compared to all other amplitudes of transmitted waves which are very small. The variation of $\left|A_{2}^{\prime}\right|$ and $\left|A_{3}^{\prime}\right|$ with angle of incidence are plotted after magnifying their original values by the multiples of $10^{12}$ and $10^{15}$, respectively.

Figures 4 and 5 show the variation of modulus values of amplitudes of various reflected and transmitted waves with respect to angle of incidence, when a longitudinal displacement wave is incident obliquely at the interface with frequency $\omega=500 \mathrm{rad} \mathrm{s}^{-1}$, (i.e., when $l_{2} \omega>>1$ ). In Figure 4, we have plotted the amplitudes of various reflected waves against angle of incidence. It can be seen that the amplitude $\left|A_{1}\right|$ and $\left|A_{2}\right|$ begin with some finite values at normal incidence. 
Thereafter, the value of amplitude $\left|A_{1}\right|$ decreases with increase in angle of incidence till $\theta_{1}=52^{0}$ and thereafter, its value increases sharply to approach to unity as $\theta_{1}$ approaches to $90^{\circ}$, while the value of amplitude $\left|A_{2}\right|$ decreases monotonically in the range $0^{0}<\theta_{1} \leq 90^{\circ}$ and attains the value zero at grazing incidence. The variation of $\left|A_{3}\right|$ and $\left|A_{4}\right|$ is similar as in the later cases. We have plotted the amplitudes $\left|A_{2}\right|$ and $\left|A_{3}\right|$ after multiplying their original values by $10^{16}$ and $10^{7}$, respectively.

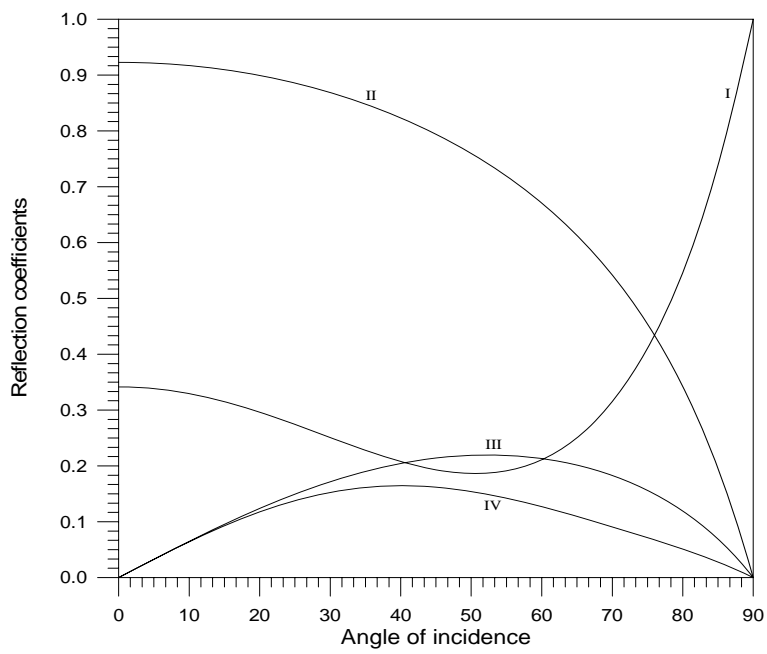

Figure 4: (High frequency case) Variation of reflection coefficients with the angle of incidence (Curve-I: $\left|A_{1}\right|$, CurveII: $\left|A_{2}\right| \times 10^{16}$, Curve-III: $\left|A_{3}\right| \times 10^{7}$, Curve-IV: $\left.\left|A_{4}\right|\right)$

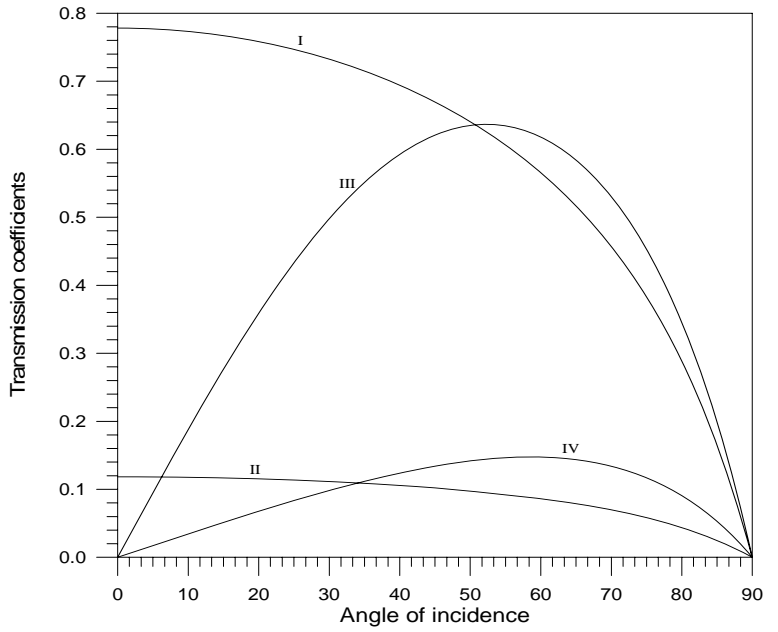

Figure 5: (High frequency case) Variation of refraction coefficients with angle of incidence (Curve-I: $\left|A_{1}^{\prime}\right|$, CurveII: $\left|A_{2}^{\prime}\right| \times 10^{15}$, Curve-III: $\left|A_{3}^{\prime}\right| \times 10^{7}$, Curve -IV: $\left.\left|A_{4}^{\prime}\right|\right)$

Figure 5 shows the variation of amplitude of transmitted waves with angle of incidence of longitudinal displacement wave traveling with speed $V_{1}$. It has been noticed from this figure that the pattern of variation of amplitudes is similar as for low frequency case as is shown in Figure 3. Here, we have magnified the values of amplitudes $\left|A_{2}^{\prime}\right|$ and $\left|A_{3}^{\prime}\right|$ by $10^{15}$ and $10^{7}$, respectively.

Numerically, it has been verified that the sum of real (imaginary) parts of the energy ratios of various reflected and transmitted waves is equal to unity (zero), at each angle of incidence at the interface, in both the cases of low and high frequency. In both the cases of low and high frequency incident longitudinal displacement wave, we note that the significant amount of incident energy goes along the reflected and transmitted longitudinal displacement waves and along the reflected and transmitted sets of coupled transverse waves at angle $\theta_{4}$ and $\theta_{4}^{\prime}$. This is because the energy carried by a wave is proportional to the square of the modulus of amplitude of that wave. Since the modulus of amplitudes of reflected and transmitted volume fractional waves and reflected and transmitted sets of coupled transverse waves with angles $\theta_{3}$ and $\theta_{3}^{\prime}$ are very small, so their squares are obviously very-very small and hence the energy carried by these waves is small enough.

\section{Conclusions}

In this paper, we have studied the phenomena of reflection and transmission of incident longitudinal displacement wave at a plane interface between two different micropolar porous elastic solid half-spaces in perfect contact. Using the appropriate set of boundary conditions, the system of simultaneous equations giving the amplitudes of various reflected and transmitted waves are obtained. The expressions of energy ratios are also presented. We conclude that

(i) The amplitudes of various reflected and transmitted waves are found to be complex valued.

(ii) The modulus of amplitudes of various reflected and transmitted waves depend upon angle of incidence, frequency and elastic properties of the medium.

(iii) Maximum amount of incident energy is carried along the reflected and transmitted longitudinal displacement waves.

(iv) The algebraic sum of the real (imaginary) parts of the energy ratios is equal to unity (zero) at each angle of incidence showing that there is no dissipation of energy at the interface during transmission. 


\section{References}

Chandrasekharaiah D. S. 1987. Effect of surface stresses and voids on Rayleigh waves in an elastic solid. International Journal of Engineering Science, Vol. 25, pp. 205-211.

Ciarletta M. and Sumbatyan M. A. 2003. Reflection of plane waves by the free boundary of a porous elastic half-space. Journal of Sound and Vibration, Vol. 259, pp. 253-264.

Cowin S. C. and Goodman M. A. 1976. A variational principle for granular materials. Zeitschrift furAngewandte Mathematik und Mechanik, Vol. 56, pp. 281-286.

Cowin S. C. and Nunziato J. W. 1983. Linear elastic materials with voids. Journal of Elasticity, Vol. 13, pp. 125-147.

Dey S., Gupta S., Gupta A. K., Kar S. K. and De P. K. 2003. Propagation of torsional surface waves in an elastic layer with void pores over an elastic half-space with void pores. Tamkang Journal of Science and Engineering, Vol. 6, pp. $241-249$.

Eringen A. C. 1966. Linear theory of micropolar elasticity. Journal of Mathematics and Mechanics, Vol. 15, pp. 909-923.

Eringen A. C. 1968. Theory of micropolar elasticity. In Fracture, Volume II, Academic Press, NY, Chapter-7, pp. 621-730.

Eringen A. C. and Suhubi E. S. 1964. Non-linear theory of simple microelastic solids-I. International Journal of Engineering Science, Vol. 2, pp. 189-203.

Goodman M. A. and Cowin S. C. 1972. A continuum theory for granular materials. Archive for Rational Mechanics and Analysis, Vol. 44, pp. 249-266.

Golamhossen F. R. 2000. Propagation of waves in an elastic cylinder with voids. Science and Technology-research Journal, University of Mauritius, Mauritius \}, Vol. 5, pp. 43-52.

Iesan D. A. 1985. Shock waves in micropolar elastic material with voids. An. Stiint. Univ. Al. I. Cuza Iasi SecI a Mat., Vol. 31, pp. 177-186.

Iesan D. and Nappa L. 2003. Axially symmetric problems for a porous elastic solid. International Journal of Solids and Structures, Vol. 40, pp. 5271-5286.

Midya G. K. 2004. On Love-type surface waves in homogeneous micropolar elastic media. International Journal of Engineering Science, Vol. 42, pp. 1275-1288.

Nunziato J. W. and Cowin S. C. 1979. A non-linear theory of elastic material with voids. Archive for Rational Mechanics and Analysis, Vol. 72, pp. 175-201.

Parfitt V. R. and Eringen A. C. 1969. Reflection of plane waves from flat boundary of a micropolar elastic half-space. Journal of the Acoustical Society of America, Vol. 45, pp. 1258-1272.

Puri P. and Cowin S. C. 1985, Plane waves in linear elastic material with voids. Journal of Elasticity, Vol. 15, pp. 167-183.

Singh B. and Kumar R. 1998. Reflection and refraction of plane waves at an interface between micropolar elastic solid and viscoelastic solid. International Journal of Engineering Science, Vol. 36, pp. 119-135.

Suhubi E. S. and Eringen A. C. 1964. Non-linear theory of simple microelastic solids-II. International Journal of Engineering Science, Vol. 2, pp. 389-404.

Tomar S. K. and Gogna M. L. 1995a. Reflection and refraction of coupled transverse and micro-rotational waves at an interface between two different micropolar elastic media in welded contact. International Journal of Engineering Science, Vol. 33, pp. 485-496.

Tomar S. K. and Gogna M. L. 1995b. Reflection and refraction of longitudinal waves at an interface between two micropolar elastic media in welded contact. Journal of the Acoustical Society of America, Vol. 97, pp. 822-830 [Erratum: in J. Acoust. Soc. Am., Vol. 102, pp. 2452 (1997)].

Tomar S. K. and Singh J. 2005. Transmission of longitudinal waves through a plane interface between two dissimilar porous elastic solid half-spaces. Applied Mathematics and Computation, Vol. 169, pp. 671-688.

Tomar S. K. and Singh J. 2006. Plane waves in micropolar porous elastic solid. International Journal of Applied Mathematics and Mechanics, Vol. 2, pp. 52-70.

Wright T. W. 1998. Elastic wave propagation through a material with voids. Journal of the Mechanics and Physics of Solids, Vol. 46, pp. 2033-2047.

\section{Biographical notes}

Dr. S.K. Tomar is a Professor in the Department of Mathematics, Panjab University, Chandigarh (UT), India. He has more than 23 years of experience in teaching and research. His current area of research includes Waves and Vibrations in continuous media, Theoretical Seismology, Numerical computations and Convection problems in Fluid Mechanics. He has published more than seventy papers in referred international journals. He visited University of Glasgow, Scotland, UK under INSA bilateral exchange programme during 2009.

Aarti Khurana is with DAV College, Sector-10, Chandigarh, INDIA

Received September 2010

Accepted March 2011

Final acceptance in revised form March 2011 